\title{
ASSESSMENT OF PCA AND MNF INFLUENCE IN THE VHR SATELLITE IMAGE CLASSIFICATIONS
}

\author{
P. C. Molina ${ }^{1,}$ M. P. Castro ${ }^{1}$, C. S. Anjos ${ }^{2 *}$ \\ ${ }^{1}$ Surveying and Cartography Engineering student, Federal Institute of Education, Science and Technology of the South of Minas \\ Gerais, Inconfidentes, Minas Gerais, Brazil - pamela_molina@ hotmail.com, maiara19castro@gmail.com \\ ${ }^{2}$ Dept. of Surveying and Cartography, Federal Institute of Education, Science and Technology of the South of Minas Gerais, \\ Inconfidentes, Minas Gerais, Brazil - camila.lacerda@ifsuldeminas.edu.br * IEEE Member
}

\begin{abstract}
KEY WORDS: Classification Accuracy, Spectral Transformations, Random Forest, Deep Learning, Remote Sensing, Satellite Imaging
\end{abstract}

\begin{abstract}
:
Orbital images have been increasingly refined spatially as spectrally as that is the case with those provided by satellite Earth observation WorldView-3 used in this paper. However, the images are very susceptible to noise interference, so it is difficult to identify and characterize objects. Therefore, it is essential to use techniques to minimize them. Thus, through increasingly innovative processing, it is possible to carry out detailed characterization mainly of urban areas. This work aims to perform the classification of images Worldview-3 using the advanced methods of classification Random Forest and Deep Learning for the region of Botafogo in the municipality of Rio de Janeiro, Brazil. Such classifications were performed for four different data sets, including the spectral bands and transformations (MNF and PCA) resulting from the original images. The results demonstrate that the use of transformations resulting from the original images as input data for the extraction of attributes in conjunction with the spectral bands improves the accuracy of the classifications generated by the Random Forest and Deep Learning method.
\end{abstract}

\section{INTRODUCTION}

Currently, there is a strong demand for remote sensing, especially for orbital images that have been increasingly enhanced by the market due to agile product and information security. This improvement is of interest to public and private companies, which are developing several projects aimed at mapping and monitoring the Earth's surface. Therefore, image classification, which is a strand of digital processing, is used for the development of these projects.

Image classification in remote sensing is the extraction of information through spectral data for recognition of homogeneous patterns and objects whose objective is the mapping of the land surface area of interest (Lock, Kirchner, 1997). The final result of this procedure is a thematic image, which is a map where the classified pixels are represented by graphic symbols or colors, which are defined by the operator. The advanced classification algorithms Random Forest (RF) and Deep Learning (DL) are usually used for the classification.

The Random Forest algorithm proposed by (Breiman, 2001), consists of a decision tree type aggregation technique, constructed in a way that its structure is composed randomly. To determine the class of an instance, the method combines the outcome of multiple decision trees through a voting mechanism. In the end, each tree gives a rating or a vote for a class. The final rating is given by the label that received the most votes among all trees in the forest (Diniz et al., 2013).

The Deep Learning algorithm is characterized by (Ponti \& Costa, 2017) as state-of-the-art in many machine-solving problems, in classification problems. It is an emerging theme within the field of artificial intelligence, which uses neural networks to improve processing results.
The difficulty found in using the Deep Learning method for image classification is the number of samples that should be collected because many times the minimum value is not achieved for all classes due to the variety of classes.

This learning uses deep layers of mathematical neurons to process data, so the information is passed through each layer in a way that the output of the previous one provides the input to the next. It is also emphasized that each of these layers is a simple and uniform algorithm containing an activation function type (Data Science Academy, 2019).

According to (INPE, 2019) the use of multispectral images is a valuable technique for extracting data intended for various research applications mainly of natural resources. Also, obtaining spectral information recorded by the systems in the different parts of the electromagnetic spectrum, aiming at the identification and discrimination of targets of interest depends mainly on the quality of data representation contained in the images.

However, even with the wealth of information of the data from multispectral sensors, it is necessary to consider the precise information, both in the characterization and in the quantification, so the extraction of image attributes is carried out. According to (Anjos, et al., 2017), this procedure aims to extract information from the dataset. These attributes are used in the classification process, since the classifiers work as data miners, identifying, in the middle of a wide set of inputs which attributes are necessary to determine the separation between the classes.

From this, characteristic extraction operations with specific purposes of processing multispectral spectral sensing

\footnotetext{
* Corresponding author
} 
information are combined, so that various aspects are explored. Therefore, different resource extraction strategies are combined, such as principal component analysis (PCA) and minimum noise fraction (MNF), so that it improves the performance of resource extraction approaches in the evaluated classification.

Aiming at the efficient separation between classes, this article aims to analyze the influence of MNF and PCA transformations on the quality of WorldView-3 multispectral image classifications, through Random Forest and Deep Learning classifiers.

\section{WORDVIEW-3 IMAGE}

The scene used in this research was acquired on May 2, 2016, corresponding to a sample of the WorldView-3 satellite over the neighborhood of Botafogo in the city of Rio de Janeiro (Figure 1). This image has been released for free by the Digital Globe platform.

It has a spatial resolution of 1.24 meters, 8 spectral bands (coastal, blue, green, yellow, red, infrared, NIR-1, NIR-2) with circular, heliosynchronous, descending orbit, $45^{\circ}$ inclination, 97 minutes period and altitude of $617 \mathrm{Km}$.

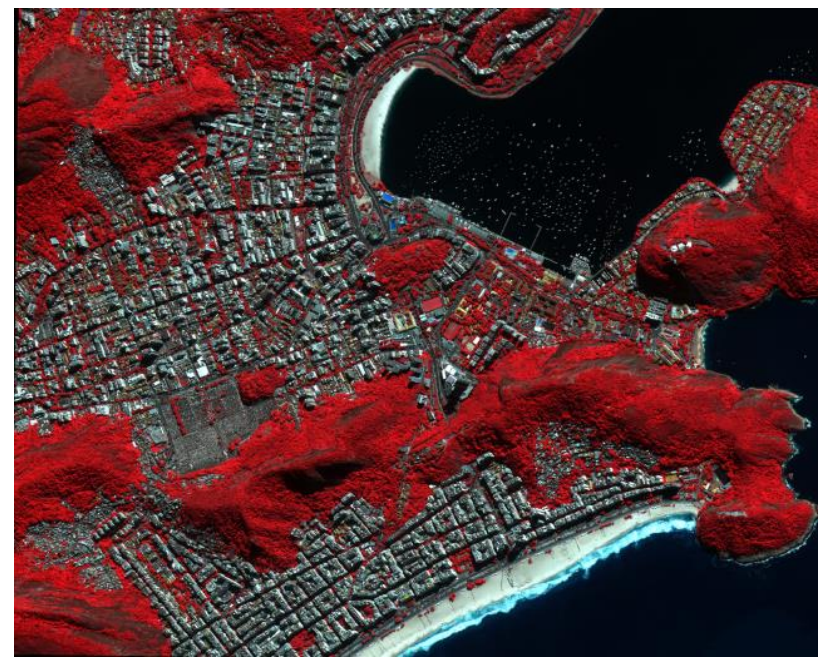

Figure 1. WorldView-3 image of study area 6R4G3B.

\section{METHODOLOGICAL PROCEDURES}

Figure 2 presents a summary of the methodology used in this work.

To perform the preprocessing was used in Envi 5.3 software. At first radiometric calibration was performed for radiance and then atmospheric correction using the tool FLAASH (Fast Lineof-sight Atmospheric Analysis of Spectral Hypercubes).

The next step was Feature Extraction to generate information from the original dataset. Main Component Analysis (PCA) and Minimum Noise Fraction (MNF) were extracted.

The Envi 5.3 software was used to perform the pre-processing. At first, radiometric calibration was performed for radiation and then atmospheric correction using the FLAASH (Fast Line-ofsight Atmospheric Analysis of Spectral Hypercubes) tool. The next step was Feature Extraction to generate information from the original dataset. Principal Component Analysis (PCA) and Minimum Noise Fraction (MNF) were extracted.

Due to the success in the first steps, the Trimble's eCognition data collection. It was used the Multiresolution Segmentation method, which aims to partition the image into homogeneous segments that present different dimensions arranged at different levels of intercommunicative segmentation with each other.

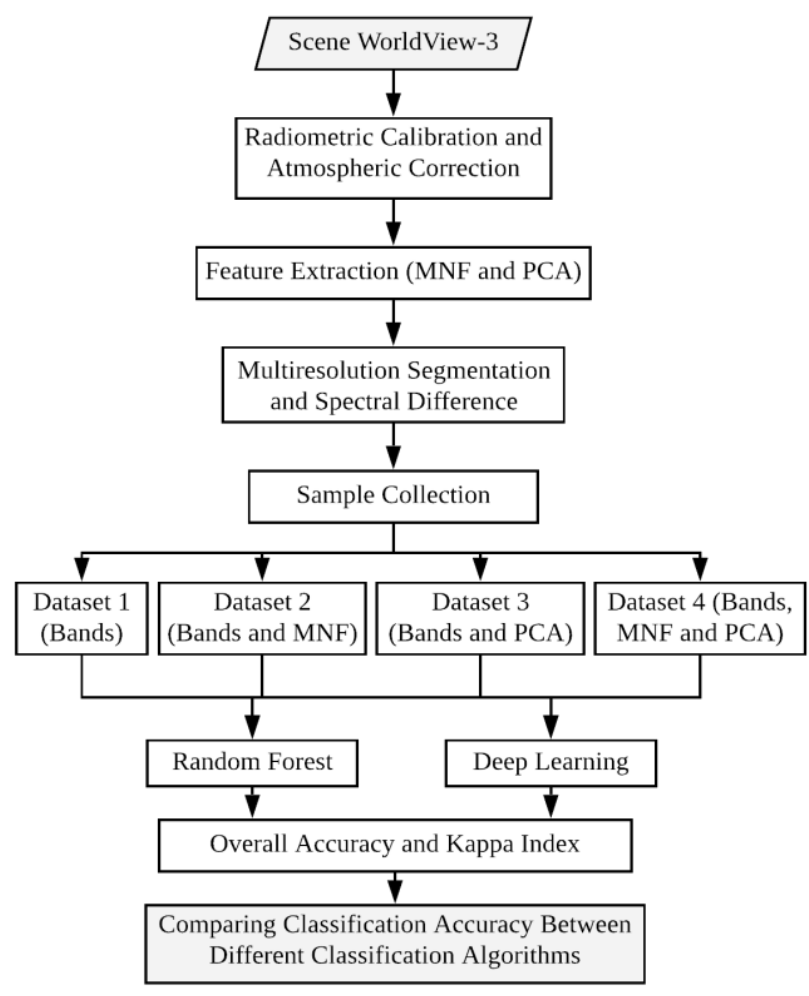

Figure 2. Methodological Procedures.

Multi-resolution segmentation allows small objects to be properly analyzed at a thinner resolution, while larger objects can be properly manipulated at a coarser resolution (Anjos, 2016). After multiresolution segmentation, it was also used the Spectral Difference algorithm, which aims to refine the targeting results through spectral similarity between neighboring segments. The following parameters were used for segmentation: scale, shape, compactness, weight and spectral difference that are presented in table 1 .

The scale parameter is assigned to obtain the average size of the segments, similarly, the influence of the shape on the degree of segmentation is observed when compared to the color. Compactness is the ratio of the length of the edge of a segment to the total number of pixels. Finally, the weight parameter must be assigned to the images according to their degree of importance in the segmentation process (varies from 0 to 1 ).

As a result, an image with small segments was obtained, but it is in accordance with the details of the scene in question.

\begin{tabular}{|c|c|}
\hline \multicolumn{2}{|c|}{ Segmentation Parameters } \\
\hline Scale & 25 \\
\hline Shape & 0.7 \\
\hline Compactness & 0.5 \\
\hline Image Layer Weights & 1 (spectral bands) \\
0 (MNF and PCA)
\end{tabular}

Table 1. Segmentation Parameters 
The next step to perform the classification was the manipulation of the CSV files in the Weka software, where the files were converted to the ARFF format to proceed in the final steps.

Data exported from eCognition has been separated into four different datasets:

- Dataset 1 containing only information from spectral bands;

- Dataset 2 containing information derived from spectral bands and MNF;

- Dataset 3 containing information derived from spectral bands and PCA;

- Dataset 4 containing the information resulting from the spectral bands, MNF and PCA.

From this, we started the procedure for the classification of datasets in the WEKA software, using the Random Forest and Deep Learning algorithms to make the comparison.

Eight classifications were generated considering the four datasets and the two classification methods (RF and DL). Besides, in the WEKA software, the eight Confusion Matrix were generated through which the Overall Accuracy and Kappa Index were calculated.

It is necessary to validate the stability of the machine learning model whose objective is to guarantee the best data standards, that is without its low bias and variance. Generally, an error estimate for the model is made after training, better known as waste assessment.

In this process, a numerical estimate of the difference in the predicted and original responses is made, also called training error. However, this provides only an idea of the model's performance in the data used to train it. Thus, there is a possibility that the model is insufficiently adjusting the data. Therefore, the problem with this evaluation technique is that there is no indication of the generalization of the independent dataset, which becomes known as cross-validation (Gupta, 2017).

Thus, to verify the data generated, we used the Weka software to use cross-validation, adjusting parameter values in the classification methods. The Kappa index tables, global accuracy and the confusion matrix are included for evaluation.

The classifications were visualized in the QGIS 3.6.1 software. in the shapefile format with the categorization of the classes defined by the classifiers that allowed the visual comparison between all datasets.

The colors defined to represent each of the 13 most relevant classes in all eight classifications are shown in Figure 3.

\begin{tabular}{|c|c|}
\hline Arboreal Vegetation & Rock \\
\hline Asphalt & Sand \\
\hline Boat & Shade \\
\hline Cemitery & Short Vegetation \\
\hline Edifications & Slum \\
\hline Bare Soil & Swimming Pool \\
\hline Ocean & \\
\hline
\end{tabular}

Figure 3. Colors determined to represent the 13 classes.

\section{RANDOM FOREST CLASSIFICATION}

Four classifications were generated with the Random Forest algorithm considering the four datasets.

Figure 4 shows the RF classification for dataset 1, which uses only spectral bands.

Analyzing the RF classification dataset 1 (figure 4) and also through the analysis of the data obtained with the classification, it is possible to perceive the confusion between classes, and boats, cemetery, edifications and slum have similarity between the spectral signatures, as they are constructed with the same material.

In addition, misconceptions are observed with the sand, rock, bare soil and shade classes. This can be justified by shape assignments, colors, and other decision-influencing patterns for classification.

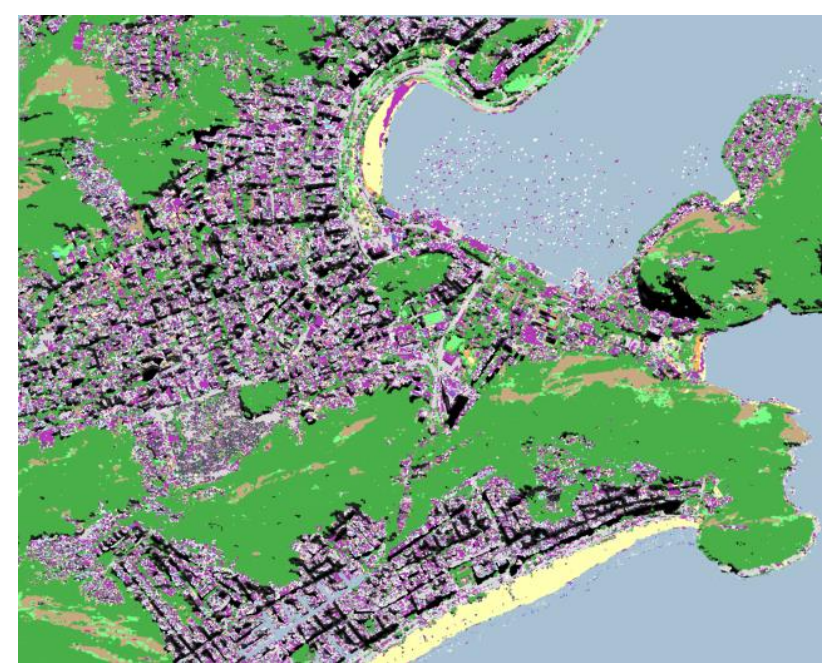

Figure 4. RF Classification for dataset 1.

Figure 5 shows the RF classification for dataset 2, which uses spectral bands and MNF.

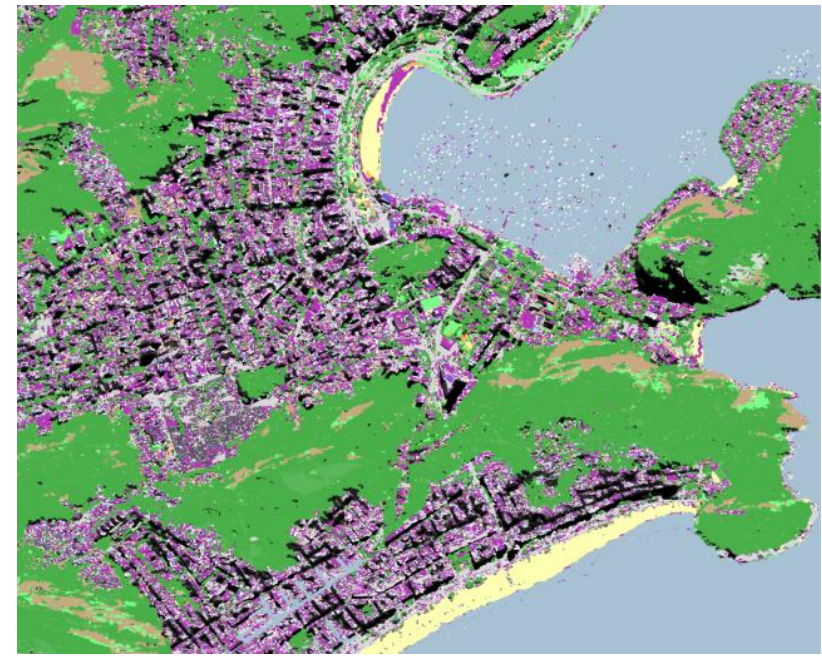

Figure 5. RF Classification for the dataset 2.

Figure 6 shows the RF classification for dataset 3, which uses spectral bands and PCA. 


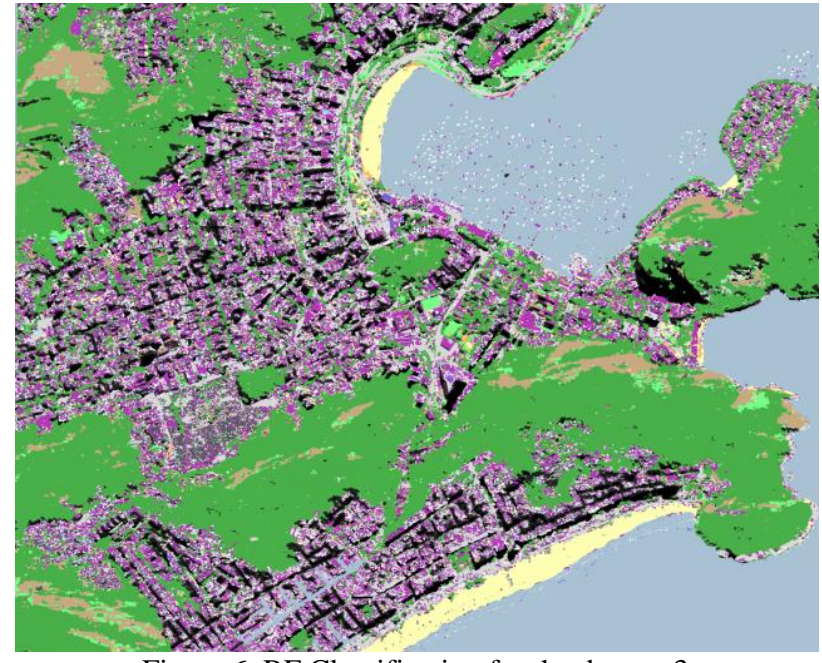

Figure 6. RF Classification for the dataset 3.

Figure 7 shows the RF classification for dataset 4, which uses spectral bands, MNF and PCA.

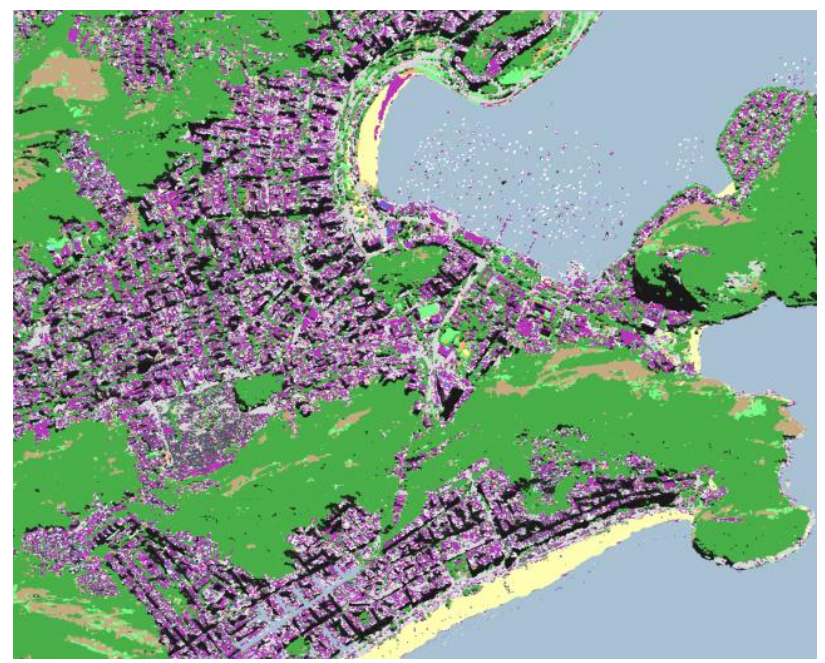

Figure 7. RF Classification for the dataset 4.

Visual analysis of the results showed no significant differences. Overall Accuracy and Kappa Index values are presented in Table 2 .

\begin{tabular}{|c|c|c|}
\hline Dataset & $\begin{array}{c}\text { Overall } \\
\text { Accuracy }\end{array}$ & Kappa Index \\
\hline $\mathbf{1}$ & $73.55 \%$ & 0.71 \\
\hline $\mathbf{2}$ & $78.75 \%$ & 0.76 \\
\hline $\mathbf{3}$ & $78.71 \%$ & 0.76 \\
\hline $\mathbf{4}$ & $79.51 \%$ & 0.77 \\
\hline
\end{tabular}

Table 2. Results of Random Forest Classification.

Analyzing the RF classification dataset 1 (figure 4) and also through the analysis of the data obtained with the classification, it is possible to perceive the confusion among classes. Boats, cemetery, edifications, and slum have similarity between the spectral signatures since they are built with the same material. Also, misconceptions are observed with the sand, rock, bare soil and shade classes. This can be justified by shape assignments, colors, and other decision-influencing patterns for classification. The same confusion continues in RF classification for datasets 2 and 3 (Figures 5 and 6), however with a smaller distribution analyzed in Figure 4.
This fact can be explained due to a small correlation between bands, without any Random Forest algorithm, according to (Anjos, 2016) works with a large collection of uncorrelated decision trees. Thus, from the original training samples, different random sample sets are created, as many sets as decision trees to allow independent trees to be created.

The result is based on the classification of each tree for a given object called a vote (Anjos, 2016). Therefore, the random forest gets a class vote from each tree and classifies each instance using the most votes.

Figures 4, 6 and 7 showed confusion between the sand and bare classes. This is because the materials have the same composition, which often differ only by granulometry (grain size).

It should be borne in mind that the reason for the confusion among classifications is due to the detail in a large number of classes and the similarity between the materials composed by each class chosen.

Note that the insertion of PCA and MNF attributes separately (datasets 2 and 3) improve the quality of the classification compared to that performed by dataset 1 .

However, the difference between the results of datasets 2 and 3 is not significant.

Inserting the PCA and MNF attributes together (dataset 4) results in the best validation indexes (Overall Accuracy and Kappa Index) when compared to all other datasets.

\section{DEEP LEARNING CLASSIFICATION}

Four classifications were generated with the Deep Learning algorithm considering the four datasets.

Figures 8, 9, 10, and 11 show the DL classifications respectively for datasets $1,2,3$, and 4 .

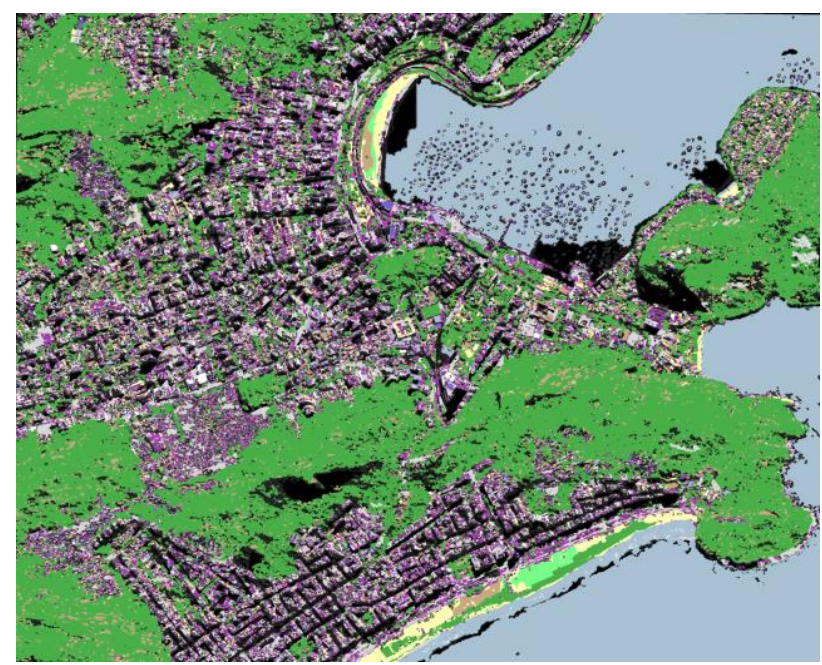

Figure 8. DL Classification for dataset 1.

The visual analysis of the results shows no significant differences. Overall Accuracy and Kappa Index values are shown in Table 3.

The classification using the Deep Learning algorithm showed significant confusion between the classes. This is due to the small number of samples collected since there is a need for data immensity to perform processing by this method. It is common 
to use this type of tool for learning in large amounts of data (Big Data).

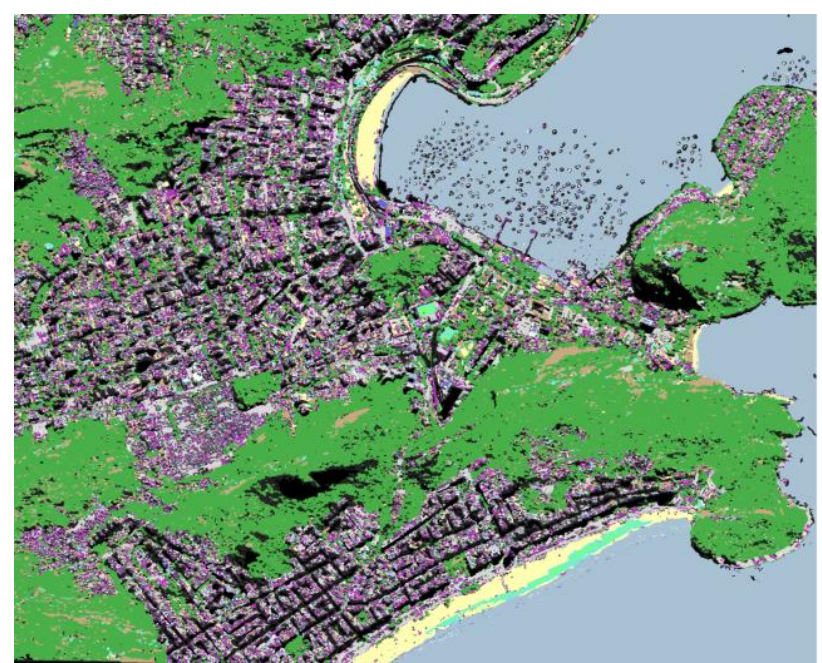

Figure 9. DL Classification for the dataset 2.

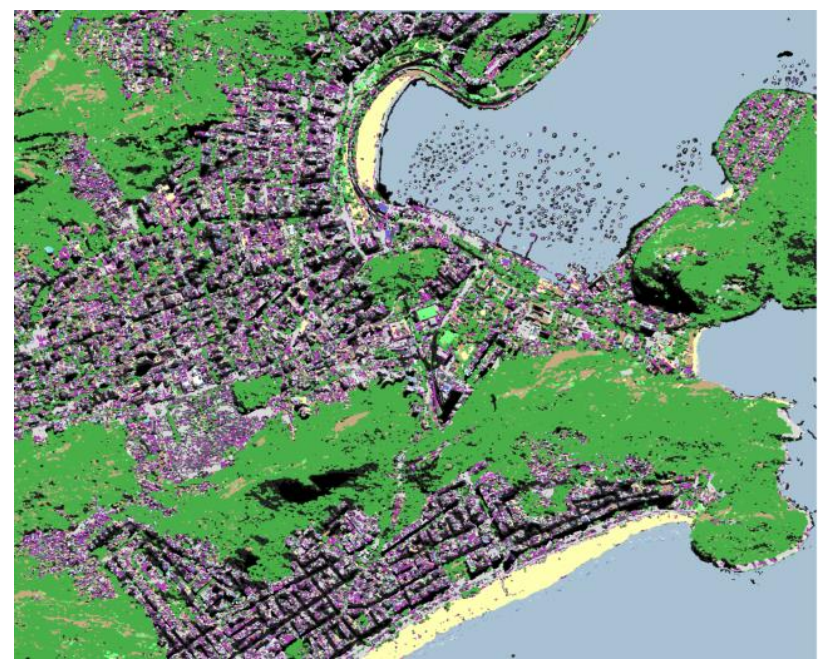

Figure 10. DL Classification for the dataset 3.

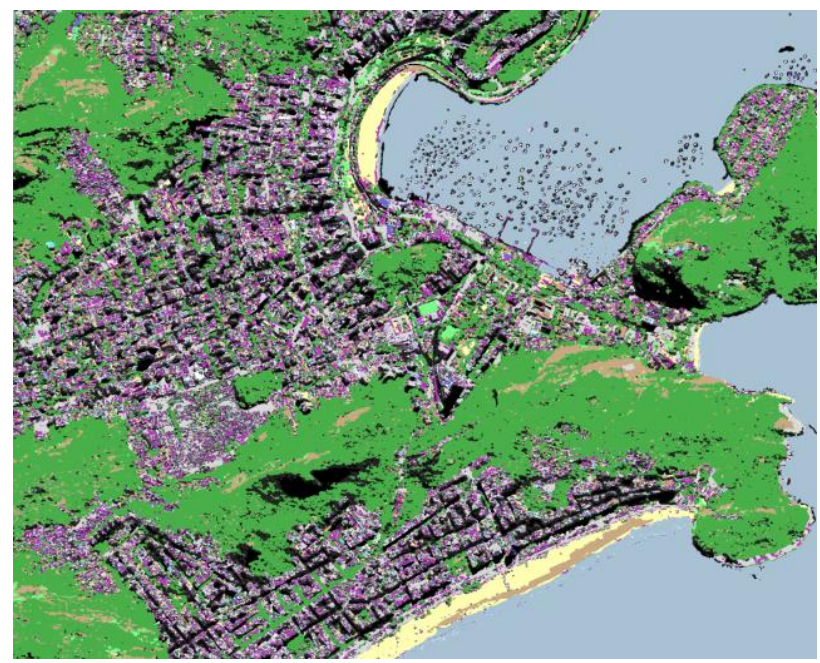

Figure 11. DL Classification for the dataset 4.

When analyzing figures $8,9,10$ and 11 , confusion scans, such as building areas that were classified as boats. Such confusions occur due to the similarity of the material used or even the color of the objects. To occur a significant separation, it would be ideal to collect a larger number of samples, but this would only be possible in a pixel-by-pixel approach, which could cause other problems in classifications such as salt and pepper noise.

The rocks are another class that showed confusion in the execution of Deep Learning presented in figures 8, 10 and 11 because the material is dispersed in areas near low vegetation, characterizing it as well as bare earth, since it has similarities in the response spectral.

In addition, just as in RF, the Deep Learning algorithm presented notable misunderstandings between the rock and sand classes. This can be justified by the training process, as they are derivable materials. Thus, (Chaves, 2019) this explains that the sand is formed from the dismantling of the rocks of some nearby mountain range, which usually takes millions of years to degrade. It adds that the geographical origin of the material may be in a mountain range a few meters/kilometers from the beach, as is the case with Botafogo beach in the municipality of Rio de Janeiro

Compared to Random Forest, Deep Learning has confused darker regions such as the surrounding buildings and the ocean. These can be seen in figure 8 , classified as shadow due to the coloration presented in the region.

Note that the insertion of PCA and MNF attributes separately (datasets 2 and 3) significantly improves the quality of the classification compared to that performed by dataset 1 . Furthermore, the insertion of the PCA and MNF attributes together (dataset 4) results in the best validation indexes (Overall Accuracy and Kappa Index) when compared to all other datasets.

\begin{tabular}{|c|c|c|}
\hline Dataset & $\begin{array}{c}\text { Overall } \\
\text { Accuracy }\end{array}$ & Kappa Index \\
\hline $\mathbf{1}$ & $54.06 \%$ & 0.49 \\
\hline $\mathbf{2}$ & $61.78 \%$ & 0.58 \\
\hline $\mathbf{3}$ & $62.06 \%$ & 0.58 \\
\hline $\mathbf{4}$ & $62.86 \%$ & 0.59 \\
\hline
\end{tabular}

\section{CONCLUSIONS}

From the results generated by the classification of the images by the advanced classifiers Random Forest and Deep Learning, it is observed that the greater the number of attributes, the better the result.

In the case of this work, from Overall Accuracy, it can be noticed a better classification based on the image set which includes spectral bands, MNF and PCA for both the Random Forest and Deep Learning algorithms.

(Zortea et al., 2004) agree with the results obtained by stating that in a classification process, accuracy typically increases as they have additional information. However, the authors are concerned about the fact that the accuracy of the classifier can reach a maximum value at a certain point, and decrease with the introduction of additional bands. This is the well-known phenomenon of Hughes.

In order to improve the quality of classifications, it is suggested the use of hyperspectral and LiDAR data to reduce confusion caused by materials that have a similar spectral signature. 


\section{ACKNOWLEDGEMENTS}

Digital Globe for acquiring and providing the data used in this study.

\section{REFERENCES}

Anjos, C. S. Classificação de áreas urbanas com imagens multiespectrais e hiperespectrais utilizando métodos nãoparamétricos. Ph.D. Thesis. São José dos Campos, 2016.

Anjos, C. S., Almeida, C. M. D., Galvão, L. S., Souza Filho, C. R., Lacerda, M. G., \& Prati, R. C. (2017). Análise do nível de legenda de classificação de áreas urbanas empregando imagens multiespectrais e hiperespectrais com os métodos árvore de decisão C4. 5 e Floresta randômica. Boletim de Ciências Geodésicas, 23(2), 371-388.

Breiman, L. (2001). Random forests. Machine learning, 45(1), $5-32$.

Chaves, D. C. (2019). Avaliação do impacto ambiental da extração de areia na microbacia do Rio Maracanã-Pa.

Data Science Academy. Deep Learning Book, 2019. Disponível em: <http://www.deeplearningbook.com.br/>. Acesso em: 10 abril. 2019.

Diniz, F. A., Neto, F. M. M., Júnior, F. D. C. L., \& Fontes, L. M. O. (2013). RedFace: um sistema de reconhecimento facial baseado em técnicas de análise de componentes principais e autofaces. Revista Brasileira de Computação Aplicada, 5(1), 4254.

Gupta, P. Cross-Validation in Machine Learning. 2017. Disponível em: <https://towardsdatascience.com/crossvalidation-inmachine-learning-72924a69872f $>$. Acesso em 20 jan.2020.

Instituto Nacional De Pesquisas Espaciais (INPE). Processamento de Imagens. Disponível em: <http://www.dpi.inpe.br/spring/teoria/realce/realce.htm>. Acesso em: 15 jun. 2019.

Lock, R. E. N., \& Kirchner, F. F. (1997). Classificação de imagens multiespectrais LANDSAT TM e feições de textura: mapeamento da cobertura da terra. Floresta, 27(1/2).

Ponti, M. A., \& da Costa, G. B. P. (2018). Como funciona o deep learning. arXiv preprint arXiv:1806.07908.

Zortea, M. (2004). Investigação sobre métodos para redução de dimensionalidade dos dados em imagens hiperespectrais. 